\title{
Toward Solving the Mysteries of the Exotic Eclipsing Binary $\epsilon$ Aurigae: Two Thousand years of Observations and Future Possibilities
}

\author{
Edward F. Guinan \& Laurence E. DeWarf \\ Department of Astronomy \& Astrophysics, Villanova University, \\ Villanova, PA 19085, USA
}

Abstract. The long period $(P=27.1 \mathrm{yr})$ eclipsing binary $\epsilon$ Aurigae (F0 Ia + disk?) is truly an exotic star. It has a deep eclipse that lasts for nearly two years. This eclipse arises as a huge, cool, flattened disk transits the F-supergiant star. Modeling of the eclipse observations shows that the disk has a radius as large as $\sim 9 \mathrm{AU}$. Infrared observations indicate that the disk is cool with temperatures between $450-1000 \mathrm{~K}$. Yet there is evidence of significant FUV emission also originating from the disk.

At present, our knowledge of the mass and luminosity of the binary is still too uncertain to distinguish between two competing models of the system. The high mass model assumes that the F0 supergiant is a normal Pop. I star with a mass appropriate for its spectral type of $M \sim 15 \mathrm{M}_{\odot}$. It is accompanied by a flattened disk companion with a slightly smaller mass. In this model the disk object is a young proto-stellar or protoplanetary disk. In the low mass model, the F0I star is assumed to be a bloated, old, solar mass post-AGB star. In this case the secondary object is an accretion disk with a mass of $4-5 \mathrm{M}_{\odot}$. This disk is a remnant of postmain sequence mass transfer that occurred within the last few thousand years. In both models there are still problems explaining the object (or objects) at the center of the disk. Candidates include a pre-main sequence object, a black hole, or a close binary.

In this paper we review the properties of $\epsilon$ Aurigae and discuss the advances in our understanding of this enigmatic star from observations made since its last eclipse in 1982-1984. With new technologies and advanced instrumentation it is possible that the physical properties of this puzzling binary star will be found during the next decade. Once found, then $\epsilon$ Aurigae and its eclipses can be used as a laboratory for exploring (and testing) current astrophysical concepts and theories that include rapid stages of stellar evolution, binary star evolution, and the structure and dynamics of large disks.

\section{Introduction: the Saga of $\epsilon$ Aurigae}

The long period eclipsing binary $\epsilon$ Aurigae (F0 Iap + IR-disk) is one of the beststudied, but most puzzling, binary stars known. The eclipsing nature of the star was discovered in the 1820s and the eclipses has been observed every $\sim 27$ years since then. The last eclipse occurred during 1982-1984 and the next eclipse 
is expected in 2009-2011. This 3rd mag binary consists of an F0 Ia supergiant and a huge mysterious infrared disk companion that eclipses the primary every 27.1 years. An extraordinary amount of data have been collected on this exotic star (especially near the time of the last eclipse), but this has led to few firm conclusions. The exact nature of the disk and what is hidden at its center is still not known with certainty. Also, the masses and evolutionary states of the binary members are not well known. In one model, the F-supergiant is a normal (massive), young Pop. I star and the disk is remnant of pre-main sequence stellar evolution. In the another model, the $\mathrm{F}$ supergiant is a bloated solar mass postAGB star and the disk is the result of accretion when the F-star was filling its Roche lobe. Both of these models have some advantages and disadvantages as will be discussed later. It has been suggested that the unseen object (or objects) at the disk's center is a pre-main sequence star, an heavily obscured hot main sequence star, a close binary system, and even a stellar black hole. In this paper we discuss the progress made in determining the properties and evolutionary status of this exotic eclipsing binary.

\section{Developing Ideas about the Disk Component}

The mystery begins with the nature of the eclipsing object. The eclipse of the F-supergiant by the disk lasts for nearly 2 years with a flat bottom that lasts for $\sim 9$ months. The flat bottomed eclipse is typically taken to indicate that the eclipse is total (i.e. a total occultation) and no light from the eclipsed object is visible. The spectrum of the system, however, remains essentially unaltered during the eclipse, and no obvious spectroscopic or photometric signatures of the eclipsing object are seen. During the deepest part of the eclipse, the system is approximately half as bright as outside eclipse. Moreover, there is no significant dependence of the depth of the eclipse except at far ultraviolet (FUV) wavelengths $(\lambda<1400 \AA)$ and the far infrared where the eclipse becomes shallower. It was recognized for nearly a century that these circumstances indicate that the cool companion must be an unusual object which blocks half of the supergiant's light, yet gives a nearly flat-bottomed eclipse while remaining invisible itself.

\subsection{Early Models}

Many different interpretations of the unseen companion have been advanced. Kuiper, Struve, \& Strömgren (1937), for example, proposed that the secondary is a huge $\left(>3000 \mathrm{R}_{\odot}\right)$, tenuous star which is partially transparent. (For much of this century, astronomy textbooks listed $\epsilon$ Aurigae as the largest star in the Universe!) Hack (1961) later modified this model by placing a hot star at the center of this tenuous sphere, thus creating a Strömgren sphere. However, it was impossible to physically justify these models. Therefore, Huang (1965) proposed that the unseen object is an opaque disk that is perpendicular to our line of sight. The disk is thick, so that it appears as a rectangle in projection - the so-called "brick" model. As it passes in front of the supergiant, it covers half of the star's area, and the eclipse is flat between second and third contact. A modified, more sophisticated version of this was given by Wilson (1971) who proposed a thin, semi-opaque disk which is slightly tilted with respect to the orbit of the system. Later observations lend support to this model. 


\subsection{Disk Properties from Eclipse Analyses}

The last eclipse of $\epsilon$ Aurigae in 1982-84 has been very well covered from ultraviolet to infrared wavelengths (see Stencel 1985). A study of the photometric and spectroscopic observations that yields more reliable determinations of the physical properties of the system has been conducted by Carroll et al. (1990). As shown in Fig. 1, the photometry obtained during the eclipse has been successfully modeled by an eclipse of the supergiant star by a large, thin, semi-opaque disk viewed nearly edge-on. As shown in the figure, the brightness of the system increases near the middle of the eclipse. This mid-eclipse brightening might at first be thought to result from an increase in luminosity of the F0I star, which is known to have small light variations due to pulsations. However, the pulsations of the supergiant are known to lead to both luminosity and color variations with the star becoming hotter and bluer as it becomes brighter (Gyldenkerne 1970; Carroll et al. 1990). As discussed by Carroll et al. (1990), no significant wavelength dependence was observed during the mid-eclipse light increase. Therefore, the mid-eclipse brightness increase is most likely a result of less light being blocked by the central region of the disk. Carroll et al. (1990) fit the eclipse, including the central brightening, with a thin disk that has a central hole and transitional semi-opaque central region. The fits to the data with different models are shown in Fig. 1. The best fit is with the tilted, thin disk model. This analysis indicates that the disk is quite large with an estimated outer radius of $\sim 9 \mathrm{AU}$, which is about 10 times the radius of the supergiant, and the radius of the transparent inner hole is nearly $10 \%$ of the radius of the disk. Also, the disk is tilted about $2^{\circ}$ relative to its orbital plane. As shown in Fig. 2, this disk would appear as a thin ellipse with a central hole, a semi-opaque transitional region, and an extensive opaque outer zone. Analysis of the eclipse photometry was also carried out by Ferluga (1990) using a concentric ring model with a central opening. This analysis leads to similar results for the shape and dimensions of the disk to those found by Carroll et al. (1990).

The first physical model of the disk was developed by Lissauer et al. (1996). Hydrostatic models of the disk appropriate for $\epsilon$ Aur reproduced the overall characteristics of the observed eclipse, including the mid-eclipse brightening. Lissauer et al. (1996) further suggest that the lack of a wavelength dependence of the eclipse indicates the disk contains particles that are significantly larger than dust grains in the ISM.

\subsection{Disk Properties from Infrared Studies}

Infrared observations of the system inside and outside eclipse indicate that the disk is dusty and cool with a mean temperature $\sim 475 \pm 50 \mathrm{~K}$ (Backman et al. 1984). More recently, infrared photometry made from the ground and with the Infrared Satellite Observatory (ISO), confirmed the IR excesses found previously and indicate disk temperatures between $400 \mathrm{~K}$ and $1000 \mathrm{~K}$ (see Taranova \& Shenavrin 2001). For example, Taranova \& Shenavrin (2001) find an IR excess at $\mathrm{M}(5 \mu \mathrm{m})$ of $\sim 0.2 \mathrm{mag}$ and at $\mathrm{N}(10 \mu \mathrm{m})$ of $0.4 \mathrm{mag}$. The higher temperature inferred for the disk at shorter IR wavelengths could arise from heating of the disk by the F-supergiant directly or kinetic heating from impacting winds. It is also possible that the inferred IR excess implies that the higher temperature originates from the inner portions of the disk. 


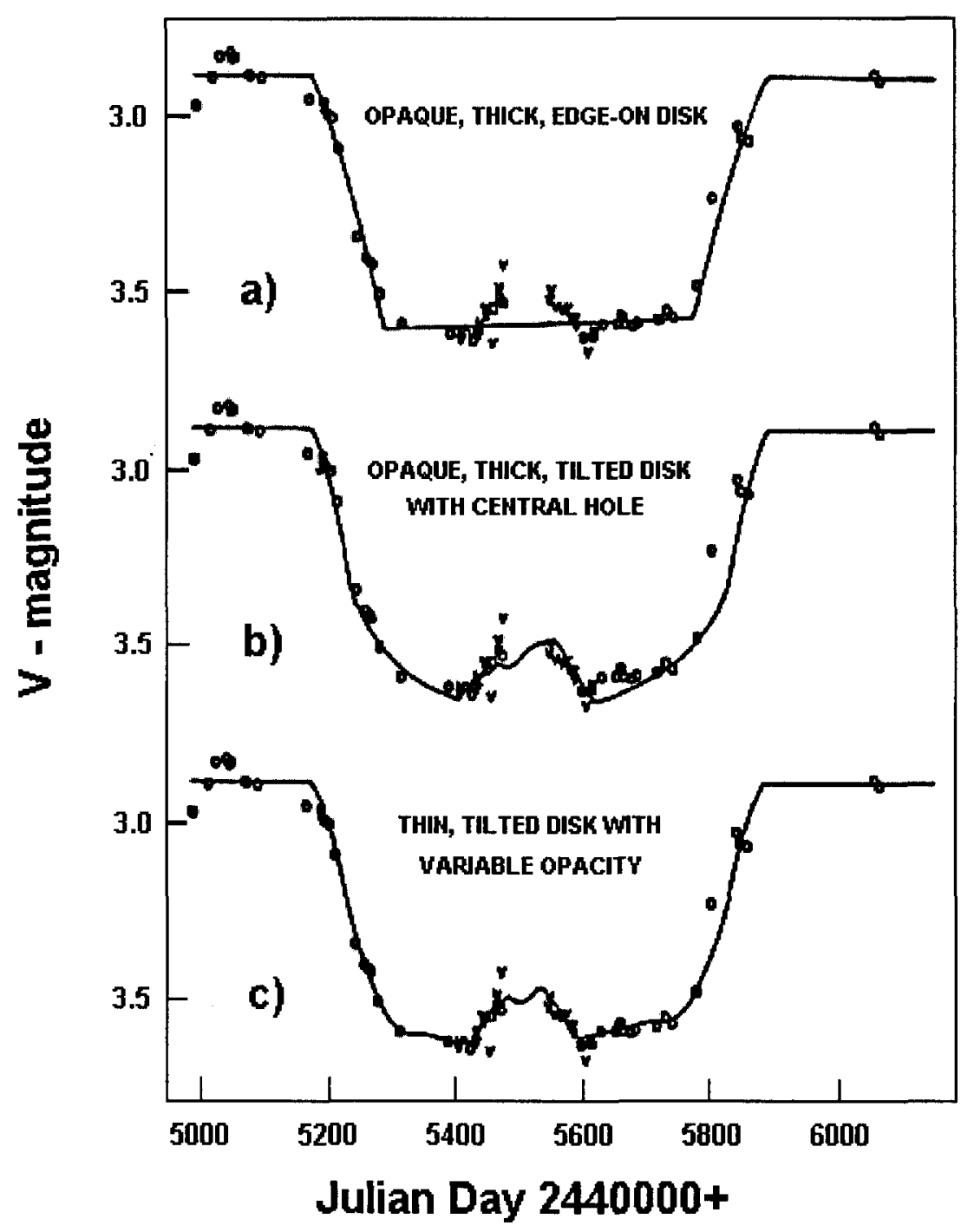

Figure 1. Modeling the 1982-84 Eclipse Light Curves of $\epsilon$ Aurigae. Theoretical and photoelectric light curves for the 1982-84 eclipse are shown. a) An attempt to fit the data with Huang's (1965) thickdisk model; this cannot account for the mid-eclipse brightening. b) A thick, tilted opaque disk with small central opening. Although this gives a good fit to the data, we felt that it did not reproduce with sufficient accuracy the flat bottom characteristic of this and previous eclipses. c) An excellent fit is achieved by using a tilted thin disk with variable opacity. The models are given by Carroll et al. (1990). 


\section{SCHEMATIC MODEL OF EPSILON AURIGAE AS SEEN NEAR MID-ECLIPSE}

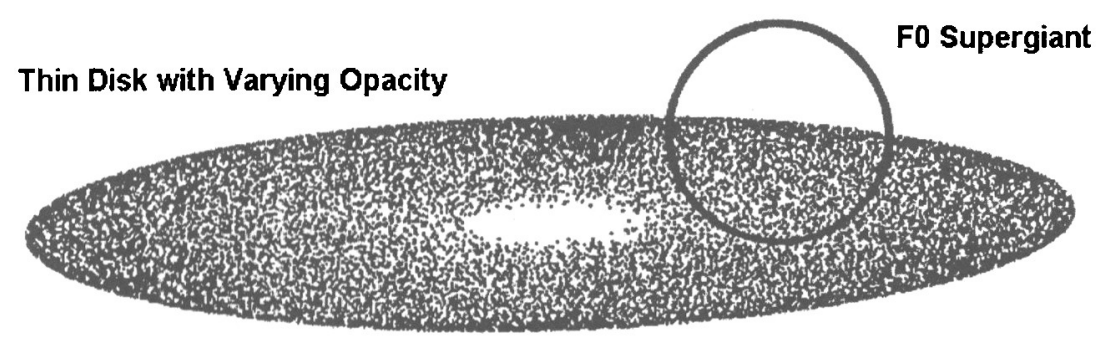

Central Hole

Figure 2. Tilted, Thin Disk Model for $\epsilon$ Aurigae. The tilted, thin disk model with a central hole and varying opacity is shown. This is the model for $\epsilon$ Aurigae adopted by Carroll et al. (1990). The disk is completely opaque near the rim, has a semi-transparent transitional region, and a completely transparent center.

Far-IR observations of the secondary eclipse (when the F0 I star transits the disk) would be very important. Unfortunately, the time of secondary eclipse is not well determined because of the uncertain values of the orbital eccentricity and the argument of periastron. Most likely times were between 1996-2001 but no IR observations of this event have been reported so far.

\section{The Nature of the Disk and What's Hidden Inside}

While our basic understanding of the morphology of $\epsilon$ Aurigae now seems more secure, there still exist many questions about the nature of the system and how this unusual configuration came to be. If the canonical interpretation of the dark companion as a disk is correct, the disk must be extremely large to cause such long eclipses; how did such a disk come to be? Is it a remnant of the formation of the system and perhaps a protoplanetary disk, or is it an artifact of recent mass transfer from the bright star? We know that the supergiant is an F0I star; however, we do not know its evolutionary history. Is it a young massive star that has recently left the main sequence and is now traveling to the right on the H-R diagram, or, as suggested by Eggleton \& Pringle (1985), an evolved 1-2 $\mathrm{M}_{\odot}$ star that is a post-asymptotic giant branch (AGB)/proto-planetary nebula (PPN) star that is evolving toward a planetary nebula phase and eventually to become a white dwarf? Supergiants are typically quite massive, and observations of the orbit of $\epsilon$ Aurigae indicate that the secondary should be comparable in mass to the primary. Since the disk itself is probably not very massive (compared to the star), the mass must primarily reside in the invisible object at the center of the 
disk. If there is a massive object at the center of the disk, why don't we see it - why is it so under-luminous?

While none of these questions may be answered definitively, the answers that have been proposed have involved many fascinating aspects of modern astrophysics. We will examine these questions in both the context of star and planet formation and stellar evolution to determine how stellar evolution theory can help us unravel the mystery of $\epsilon$ Aurigae and how observations of this unique system can help us test and refine our theories. To constrain the possible scenarios of $\epsilon$ Aurigae's evolution, we briefly review the relevant observational data and derived facts. Considering these facts leads to two general possibilities, one in which the star is very massive and the supergiant has just evolved off the main sequence (the "high-mass" model) and a more recent suggestion that the star is less massive and more evolved. We then consider these two scenarios in detail, discussing the different ways in which they could be viable, and what makes them important for stellar evolution. Finally, we discuss a problem which plagues both models: the nature of the unseen object(s) at the center of the disk. We conclude by drawing some conclusions and suggesting several provocative questions that remain to be answered as well as new observations that may help solve the mysteries of $\epsilon$ Aurigae.

\subsection{Constraining the Properties of $\epsilon$ Aurigae}

The determination of an accurate parallax for $\epsilon$ Aurigae would permit the absolute magnitude of the F0 I star to be measured and the properties and evolutionary state of the binary to be better constrained. With an accurate distance and absolute magnitude of the F0I star, the nature of the F star (normal high mass supergiant or low mass post-AGB star) could be distinguished. For example the expected absolute magnitude of a Pop. I helium burning F0 I star is listed in Allen's Astrophysical Quantities (4th ed.) as $\mathrm{M}_{V}=-6.6 \mathrm{mag}$ (Drilling \& Landolt 2000). However, other sources list absolute magnitudes of F-supergiants as large as $M_{V}=-8 \mathrm{mag}$. On the other hand post-AGB supergiants are less luminous and typically are expected to have absolute magnitude values between $\mathrm{M}_{V} \sim-4$ to $-6 \mathrm{mag}$ (Iben 1982).

From the F0 Ia spectral classification of the luminous component and $\left(\mathrm{m}_{V} \sim\right.$ $+3 \mathrm{mag}$ ), $\epsilon$ Aurigae is expected to be at a distance $d>500 \mathrm{pc}$ and thus should have a trigonometric parallax too small to be measured reliably from the ground $(\pi<2 \mathrm{mas})$. Ground-based parallax measures range from $-1.0<\pi<6.0$ mas. However, it was hoped that the Hipparcos mission would finally provide the important measurement of the trigonometric parallax of $\epsilon$ Aurigae. The parallax found from Hipparcos is $\pi$ (Hipp) $=1.60 \pm 1.16$ mas that corresponds to a distance $d=625 \mathrm{pc}$. Although the precision of the Hipparcos parallax is far better than any trigonometric parallax previously secured for $\epsilon$ Aurigae from the ground, the star is too far away for an accurate parallax measure. At face value the measured Hipparcos parallax limits permit a range of distances $450 \mathrm{pc}<d<850 \mathrm{pc}$. These distance limits correspond to absolute magnitudes of the F0I star that range from $M_{V}=-6.1 \mathrm{mag}$ to $M_{V}=-7.4 \mathrm{mag}$ when the interstellar absorption from Morris (1962) of $\mathrm{A}_{V}=0.84 \mathrm{mag}$ is adopted. As discussed by Carroll et al. (1990), most of the indirect distance estimates (spectroscopic, pulsation theory, membership in an $\mathrm{OB}$ association, etc.) indicate greater distances, up to $1.3 \mathrm{kpc}$. 
The Hipparcos parallax is in good accord with the dynamic parallax derived from equating the measured astrometric angular semimajor axis of the F0 I star's orbit with the spectroscopic (radial velocity) semimajor axis. Heintz \& Cantor (1994) have analyzed 44 years of Sproul Observatory Plates and calculated an orbital semiamplitude of $\alpha_{1}=22.4 \pm 1.8$ mas which yields a parallax of $1.65 \pm 0.15$ mas. This result is in excellent agreement with the previous orbital astrometric study carried out by van de Kamp (1978). The agreement between the astrometric parallax and the Hipparcos trigonometric parallax could be fortuitous but these measures now place more confidence in adopting a distance of $d=625 \mathrm{pc}$ and $\mathrm{M}_{V}=-6.8 \mathrm{mag}$ for the F0I star.

The other important stellar parameter which is uncertain at present is the mass of each component. Morris (1962) has determined the mass function of the system to be $f(m)=3.12 \mathrm{M}_{\odot}$. Therefore, knowing the mass of the primary would determine the mass of the secondary as well since the orbital inclination is known. Usually the mass of the primary is taken to be approximately $15 \mathrm{M}_{\odot}$, a value consistent with the most common spectral classification F0 Ia and an absolute magnitude of $\mathrm{M}_{V} \sim-7.0 \mathrm{mag}$. In this case, the mass of the secondary becomes approximately $13 \mathrm{M}_{\odot}$, comparable to that of the primary. More recently, however, Eggleton \& Pringle (1985) have suggested that the bright star might be a post-AGB star with a mass as low as $1.0 \mathrm{M}_{\odot}$. The secondary would then be about $5 \mathrm{M}_{\odot}$.

More importantly, however, the choice between the two models defines the evolutionary history of the system as well as its dimensions. Reconstructing the evolution of $\epsilon$ Aurigae involves interesting and important concepts in stellar theory, including very different physics for the two models. Therefore we should scrutinize these proposals with care, determining what the system is like, how it got to be that way, and what we can hope to learn if one or the other model is correct.

\subsection{High-Mass Model - The Case for a Protoplanetary Disk in $\epsilon$ Au- rigae}

Most F0 I supergiants are thought to be stars which have recently left the main sequence, and are now in a helium shell burning phase of evolution. Such stars typically have masses near $\sim 15 \mathrm{M}_{\odot}$ and luminosities $\log L / L_{\odot} \sim 4.5-5.0$, although considerable variation is possible. These values are consistent with the observational constraints on the $\epsilon$ Aurigae primary, so this is the evolutionary state that has usually been assigned to the star. The mass of the cool object, as has already been mentioned, is $\sim 13 \mathrm{M}_{\odot}$ with a mass function $f(m)$ of $3.12 \mathrm{M}_{\odot}$. From the duration of the eclipse, Carroll et al. (1990) find that the radius of the disk is approximately ten times that of the star. They adopted a nominal radius of the F0 Ia star of $200 \mathrm{R}_{\odot}$ and this results in a disk radius $R\left(\right.$ disk) $\sim 2000 \mathrm{R}_{\odot} \sim$ $9.3 \mathrm{AU}$, and the radius of the central hole is $\sim 0.7 \mathrm{AU}$. Kepler's third law yields a semi-major axis of $a=27.6 \mathrm{AU}=5930 \mathrm{R}_{\odot}$. This model from Carroll et al. (1990) is shown in Fig. 3. As shown, the outer dimensions of the disk are nearly the size of Saturn's orbit. As shown in the figure, these calculations immediately yield an interesting result: while the disk is close to its Roche lobe, the F-star is well inside its Roche lobe. If the assumed evolutionary history of the primary is correct, moreover, the star is as large now as it ever has been. This means 


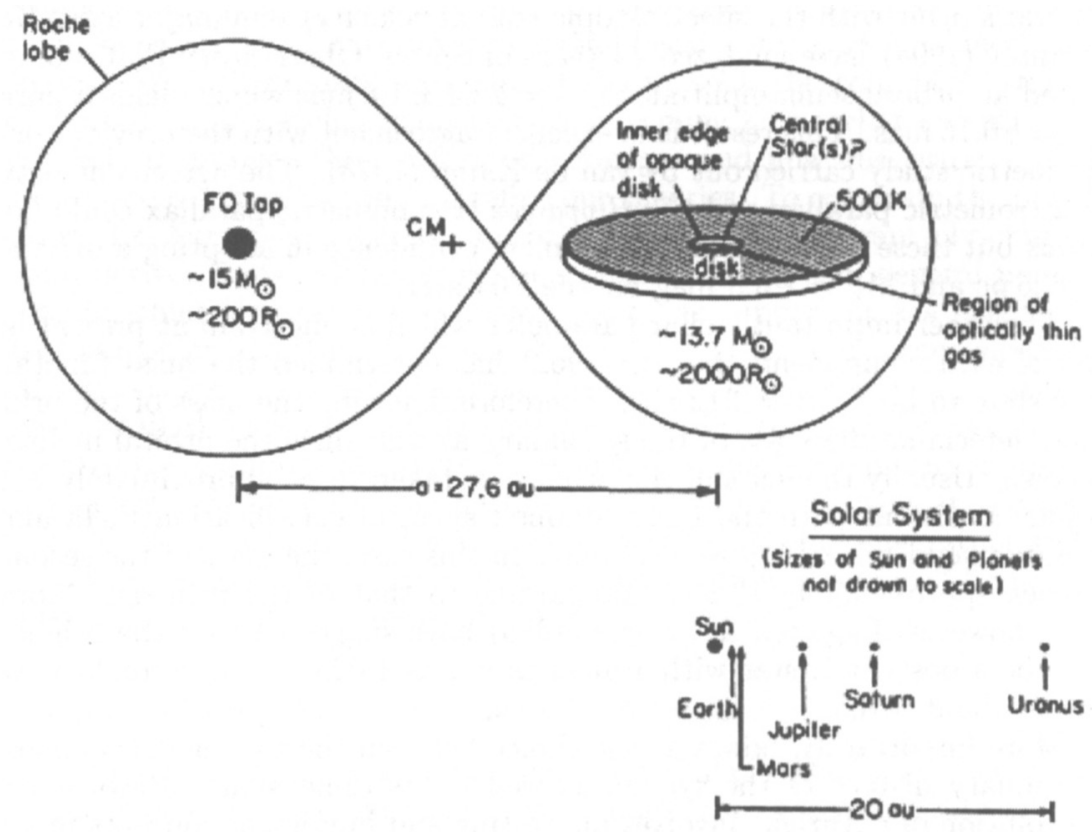

Figure 3. The scale model of $\epsilon$ Aurigae from Carroll et al. (1990) is shown. This model is appropriate for the case in which the F-supergiant is assumed to be a helium shell burning Pop. I supergiant. If the supergiant is assumed to be a low mass post-AGB star, the size of the orbit is scaled down so that the orbital semimajor axis $a=17 \mathrm{AU}$. In the low mass case, the mass of the supergiant is about $1.0 \mathrm{M}_{\odot}$ and the disk (and binary stars at its center) have a mass of $4-5 \mathrm{M}_{\odot}$.

that the disk is most likely not a result of the primary overflowing its Roche lobe and transferring mass to the secondary. Furthermore, while the spectral features of the primary (Castelli 1978) do show P Cygni profiles which indicate mass loss $\left(d M / d t \sim 10^{-7} \mathrm{M}_{\odot} / \mathrm{yr}\right)$, this seems to be much too small to result in the presence of the huge disk we observe. This may be somewhat distressing, since disks in binary systems are almost always thought to result from mass transfer. In the high-mass model of $\epsilon$ Aurigae, however, this possibility seems to be ruled out; the disk must be a remnant of the formation of the system. In this case, the disk could represent a protoplanetary disk, as suggested previously by Kopal (1971), Webbink (1985), and Carroll et al. (1990).

How feasible is it that the disk remains from the formation of the system? From stellar evolution calculations, a $15 \mathrm{M}_{\odot}$ star is expected to reach the F0 supergiant stage at an age of approximately $10^{7} \mathrm{yr}$. (Iben 1967; Lamb, Iben, \& Howard 1976). It is quite plausible for a large disk to remain intact for 
this long. It is well known, of course, that such protoplanetary disks appear to be common in young stars up to an age of at least $10^{7}$ years (see Strom et al. 1989a,b); and evidence has been found for extremely large (protoplanetary) disks around the nearby main sequence A-type stars such as $\alpha$ Lyrae (Vega), $\alpha$ Piscis Austrinis (Fomalhaut) and $\beta$ Pictoris (see Smith \& Terrile 1984; Paresce $\&$ Burrows 1987). The large size of $\epsilon$ Aurigae's disk is well within the bounds of protoplanetary disk dimensions; it is somewhat smaller than the solar system, as well as the $400 \mathrm{AU} \beta$ Pictoris disk (Smith \& Terrile 1984). (In fact, if the disk is protoplanetary, it is plausible to imagine that it would have been much larger if it weren't for the Roche lobe caused by the proximity of the supergiant star.) Furthermore, Paresce \& Burrows (1987) show that the disk around $\beta$ Pictoris is made up of grains larger than one micron, the typical size of interstellar grains. Such a composition would be able to let light from the supergiant shine through the disk during the eclipse without resulting in significant optical absorption lines, which are not observed. An extra bonus of this scenario is a natural explanation for the semi-transparent nature of the inner regions of the disk. Backman et al. (1992) have deduced from IRAS, ground-based IR $(5 \mu \mathrm{m})$, and from multi-aperture photometry that the disk around $\beta$ Pictoris has a dustfree central hole much like that inferred for $\epsilon$ Aurigae, but larger. In addition, recent infrared studies of pre-main sequence stars by Skrutskie et al. (1990) indicate that $\sim 10 \%$ of the stars sampled that have disks show evidence of inner holes. The presence of these holes is inferred from the spectral distribution of the infrared radiation. Skrutskie et al. (1990) have suggested that these holes could be produced by the formation of relatively massive planets, which keep the inner disks clean of significant amounts of matter. Alternately, energy from the pre-main sequence star or the boundary layer region could heat the inner regions of the disk, evaporating or blowing away the particulate matter.

Spectroscopic observations of young pre-main sequence (PMS) stars with disks indicate large outflows (winds) as well as infalling matter and the presence of jets and large bi-polar molecular flows (see Snell \& Edwards 1981). Also excess continuum ultraviolet and optical radiation have been discovered from some of these PMS objects and have been interpreted as arising from the inner regions of the disk (the boundary layer) where matter is secreting onto the central star.

$\epsilon$ Aurigae shows evidence of mass outflows from the P Cygni-type line profiles seen in its spectrum. However, almost all luminous supergiants have P Cygni profiles indicating large mass loss. On the other hand Carroll et al. (1990) report a significant increase in $\mathrm{H} \alpha$ absorption near the middle of the primary eclipse. This additional hydrogen absorption is observed in both the $\mathrm{H} \alpha$ photometry and directly with high resolution $\mathrm{H} \alpha$ spectroscopy. This additional $\mathrm{H} \alpha$ absorption appears to arise from gas in the central regions of disk when it is seen projected against the supergiant. It is not implausible that gas is flowing out of the central region of the disk analogous to the outflows observed in many pre-main sequence stars with disks. Furthermore, the infrared and ultraviolet properties of $\epsilon$ Aurigae are also consistent with the protoplanetary disk hypothesis. The infrared studies of $\epsilon$ Aurigae are consistent with the presence of large dust grains, while in the ultraviolet there appears to an unexplained ultraviolet excess (Boehm et al. 1984). Thus, a natural explanation for the disk in $\epsilon$ Aurigae is that it is a protoplanetary/protostellar disk, similar to those inferred for pre-main sequence stars or the disk-like structures discovered around $\beta$ Pictoris 
and a couple of other A-type stars. The huge size, low temperature, morphology, and energy distribution of $\epsilon$ Aurigae's disk are compatible with it being a protoplanetary object. Moreover, $\epsilon$ Aurigae has the spatial and kinematic characteristics of a young (Pop. I) star. Carroll et al. (1990) find that the star is within $20 \mathrm{pc}$ of the galactic plane and has $U V W$ space motions (relative to the Local Standard of Rest in units of $\mathrm{km} / \mathrm{s}$ ) of $U^{\prime}=-4, V^{\prime}=-9$, and $W=0$. A comparison of the properties of $\epsilon$ Aurigae's disk with those of protoplanetary disks is given in Table 1. The protoplanetary disk scenario for $\epsilon$ Aurigae appears promising but reeds much more study to be proven.

It is easy to see why the protoplanetary scenario is of interest to star and planetary formation studies: not only do we have a protoplanetary system such as that observed around other stars, but it is in an eclipsing binary, which means that every 27 years an eclipse occurs in which the light of the supergiant shines through the disk, allowing us to analyze its nature closely. Furthermore, the mid-eclipse brightening that was so prominent in the most recent eclipse was much less so in previous eclipses (see Gyldenkerne 1970); this may be an indication of evolution in the disk itself, offering us a unique opportunity to view the changing structure of a protoplanetary system.

Nevertheless, there are difficulties with this interpretation. Most significantly, at the center of the disk lurks a $\sim 13 \mathrm{M}_{\odot}$ object whose spectrum is not observed at any wavelength, even during eclipse. If the center of the disk contained a $13 \mathrm{M}_{\odot}$ main-sequence star, it would certainly be visible, even against the bright contribution of the supergiant; why is it so under-luminous? A possible way out of this and other problems is the suggestion by Lissauer \& Backman (1984) that a close binary system is embedded within the disk. They note that distributing the $\sim 13 \mathrm{M}_{\odot}$ of the secondary equally between two stars results in an object only $\sim 10 \%$ as luminous as a massive single star. Such a configuration would be stable if the close binary had a separation of $<5 \mathrm{AU}$ (Pendleton \& Black 1983). If $\epsilon$ Aurigae is young, then the proposed close binary inside the disk could consist of protostars or be a protobinary system itself.

One intriguing possibility suggested by Webbink (1985) is that the binary is extremely young $\left(\tau<10^{5} \mathrm{yr}\right)$, and still approaching the main sequence. However, the surface gravity expected for a pre-main sequence F0 star would place it in luminosity class Iab or Ib, not Ia as is observed. Other possibilities include less massive stars in stages of shell hydrogen burning or core helium burning. However, such stars also are expected to be in a slightly lower luminosity class than is observed for $\epsilon$ Aurigae. The one possibility which is not ruled out by such considerations is that the $\mathrm{F}$ supergiant is a low mass $\left(1-2 \mathrm{M}_{\odot}\right)$ post-asymptotic giant branch (AGB) star, which we now consider.

\subsection{Low-Mass Model - The Case for $\epsilon$ Aur Being a Post-AGB/PPN Object}

It was the problem of the high mass and low luminosity of the cool component which led Eggleton \& Pringle (1984) and Lissauer \& Backman (1984) to propose that $\epsilon$ Aurigae is less massive than previously assumed. They propose that the F0 supergiant component is not a normal massive young star, but a $\sim 1 \mathrm{M}_{\odot}$ star that has evolved beyond the asymptotic giant branch (AGB) and is now a postAGB star/protoplanetary nebula (PPN) object. If the mass of the supergiant is 
Table 1. Comparison of the disk component of $\epsilon$ Aurigae with Protostar/Protoplanetary disks

\begin{tabular}{|c|c|c|}
\hline Property & $\begin{array}{l}\text { Protostar/ } \\
\text { Planetary Disk }\end{array}$ & Disk of $\epsilon$ Aurigae \\
\hline \multicolumn{3}{|l|}{ Disk Dimensions: } \\
\hline $\begin{array}{l}\text { a) Central Core } \\
\text { (= Protostar) }\end{array}$ & $\sim 1-10 R_{\odot}$ & $?$ \\
\hline $\begin{array}{l}\text { b) Inner Dust-Free Region } \\
\text { (= Central Holes) }\end{array}$ & $\begin{array}{l}<1 \mathrm{AU} \\
\sim 0.7 \mathrm{AU}\end{array}$ & $\sim 0.7 \mathrm{AU}$ \\
\hline $\begin{array}{l}\text { c) Opaque Dusty Disk } \\
\text { (outer radius) }\end{array}$ & $\begin{array}{c}7-400 \mathrm{AU} \\
\text { typical } 30-100 \mathrm{AU}\end{array}$ & $\begin{array}{l}\sim 9 \mathrm{AU} \\
\text { (size may be limited } \\
\text { by presence of } \\
\text { luminous companion } \\
\text { and Roche lobe } \\
\text { radius) }\end{array}$ \\
\hline $\begin{array}{l}\text { d) Dusty Envelope/ } \\
\text { Molecular Envelope } \\
\text { or Gas Disk }\end{array}$ & $4000-7000 \mathrm{AU}$ & $?$ \\
\hline Shape of Disk & $\begin{array}{c}\text { flattened disk } \\
10 \% \text { of stars show } \\
\text { evidence of central } \\
\text { holes }\end{array}$ & $\begin{array}{l}\text { thin disk with central } \\
\text { hole; tilted } \sim 2^{\circ} \\
\text { relative to orbit }\end{array}$ \\
\hline Composition & $\begin{array}{l}\text { dust grains } \\
r \geq 1 \text { micron }\end{array}$ & $\begin{array}{l}\text { dust grains }+ \text { gas } \\
\quad r \simeq 1 \text { micron }\end{array}$ \\
\hline Temperature & $\simeq 50-200 \mathrm{~K}$ & $450-1000 \mathrm{~K}$ \\
\hline Mass (Disk) & $\simeq 0.1-0.01 \mathrm{M}_{\odot}$ & $\sim 0.1-1.0 \mathrm{M}_{\odot}(?)$ \\
\hline Radio Emission (mm) & yes & yes $(9 \mathrm{mJy}$ at $250 \mathrm{GHz})$ \\
\hline Infrared Excess & yes & yes \\
\hline $\begin{array}{l}\text { UV Excess } \\
\text { from boundary layer of } \\
\text { central star }\end{array}$ & yes & $\begin{array}{l}\text { yes, but location uncertain } \\
\text { UV excess } \lambda<140 \mathrm{~nm}\end{array}$ \\
\hline Evidence of Outflows & yes & $\begin{array}{l}\text { yes, but may be due } \\
\text { to Supergiant Companion }\end{array}$ \\
\hline Bipolar Flows & yes & maybe \\
\hline Age & $\sim \underset{1-100 \mathrm{Myr}}{\text { young }}$ & $\begin{array}{l}\text { young } \\
\sim 10 \mathrm{Myr}\end{array}$ \\
\hline
\end{tabular}


$\sim 1 \mathrm{M}_{\odot}$, then the cooler disk component would have a mass of $\sim 5 \mathrm{M}_{\odot}$ instead of the $\sim 13 \mathrm{M}_{\odot}$ by the mass function required in the high mass model. Also, all of the absolute dimensions of the system then correspondingly decrease. Then the disk would have radius of $\sim 7.0 \mathrm{AU}$. The semi-major axis of the orbit is $17 \mathrm{AU}$.

The post AGB/PPN stage of stellar evolution is very brief for a star as luminous as $\epsilon$ Aurigae $\left(\sim 10^{3}-10^{4} \mathrm{yr}\right)$ according to Iben (1982) and Schönberner (1983). The rapidity of the evolution of some post-AGB stars has been recently demonstrated by the rapid changes in brightness and color of FG Sge and Sakurai's Object. These stars have undergone large changes in brightness and temperature on time scales of decades (e.g. Herbig \& Boyarchuk 1968; Gonzalez et al. 1998; Lawlor \& MacDonald 2002; Kerber et al. 1999). However, several mostly high-galactic latitude F and G supergiants, the so-called $89 \mathrm{Her}$ stars, have been suggested as being PPN objects (Bond et al. 1984; Hrivnak et al. 1989). The majority of these PPN candidates have high space velocities and low metal abundances indicating that they are old disk or even halo objects (see Hrivnak et al. 1989). Eggleton \& Pringle (1985) suggest that the disk of $\epsilon$ Aurigae formed recently when the supergiant filled its Roche lobe and transferred mass to its companion. Like Lissauer \& Backman (1984), Eggleton \& Pringle (1985) believe that the secondary star is actually a binary system, making $\epsilon$ Aurigae a triple system.

Although this reconstruction of $\epsilon$ Aurigae's history differs remarkably from the previous model, it is equally exciting from a stellar evolution point of view. Little is known about post-AGB, protoplanetary nebula (PPN) evolution of moderate mass stars, and this system may represent a unique opportunity to observe one in action. However, the expected short life times and rarity of such stars causes us to ask how likely it is that this system, already remarkable for other reasons, should contain a PPN object. Is there any observational evidence for such an interpretation? Saito \& Kitamura (1986) claim that such evidence can be found in the decreasing size of the primary star. They have performed radial velocity studies which indicate that the F0I star has undergone episodes of catastrophic contraction, and argue from changes in contact times of the eclipse that the supergiant has decreased by $16 \%$ of its radius in the last 27 years. If this were true, it would dramatically confirm the hypothesis that the bright star is rapidly contracting. However, it seems unlikely that such a well-observed star could shrink so dramatically so quickly without producing noticeable luminosity and color changes. A further caveat is suggested by the well-known fact that the primary star pulsates (see Carroll et al. 1990). This could cause spurious radial velocity determinations and perhaps mimic a collapse of the atmosphere. While this evidence is interesting, it does not represent a compelling confirmation of the low-mass model.

Because the lifetime of a post-AGB/PPN star of high luminosity is very brief, the disk of $\epsilon$ Aurigae should have formed quite recently when the AGB star underwent a large mass-loss episode. During the mass ejection episode and shortly afterwards, the total luminosity of the binary might be expected to be significantly higher than today. To investigate this possibility, we examined star catalogues from antiquity that contain visual estimates of $\epsilon$ Aurigae. These historic magnitude estimates were obtained from the compilations made by Bailey (1843), Flammarion (1882), Peters \& Knobel (1915), and Knobel 
(1917). These catalogs include the Hipparcos Star Catalog as given in the Almagest by Ptolemy (c. 130 B.C.) and later catalogs by Al Sufi (960 A.D.), Ulugh Beg (1437), Tycho Brahe (1590), Hevelius (1603), Flamsteed ( 1700), Piazzi $(\sim 1800)$, Schmidt/Argelander (1840-1880), and Flammarion (1880). These historic records indicate that $\epsilon$ Aurigae has been near its present brightness since at least the time of Hipparchos ( 130 B.C.). Although the early magnitude estimates are not very precise (with errors of $\pm 0.5 \mathrm{mag}$ ), they do not indicate that a major change in the star's brightness has occurred. It is possible, however, that a change in brightness may have occurred, but it was not recorded. These observations do not lend support to the low mass model. However, they also do not rule it out because a significant brightness change of the star could have gone unnoticed or occurred prior to recorded history. It should be mentioned, that no significant brightness changes are expected for the high mass model over the last $10^{4}-10^{5} \mathrm{yr}$.

Another important reason for doubting the low mass AGB/PPN model of $\epsilon$ Aurigae is that the supergiant component appears to be a normal, luminous extreme Pop. I star with no apparent abundance anomalies. For example, no unusually high abundance of CNO processed matter, expected from the dredgeup pulses for a star that has passed through AGB evolution (see Iben 1982), are seen in the spectra of $\epsilon$ Aurigae. In addition, its location close to the galactic plane $\left(b \approx 1^{\circ} ; z \approx 10-20 \mathrm{pc}\right)$ and its low space velocity $(S \leq 10 \mathrm{~km} / \mathrm{s})$ indicate that it is most likely a very young object.

\subsection{What's Inside the Disk?}

Almost simultaneously with the proposal of the low-mass model by Eggleton \& Pringle (1985), Lissauer \& Backman (1984) also proposed that the secondary is a binary embedded within a disk, but in the context of the usual high-mass model. Dynamically, there are advantages and disadvantages to the proposal. An advantage is that orbital resonances with the period of the primary, similar to those which confine Saturn's rings, may serve to define the apparent sharp outer edge of the disk as well as the presence of the inner hole. An additional benefit is that the close binary could transfer angular momentum to the inner disk, keeping the disk itself stable for a longer time scale than would otherwise be expected.

A possible disadvantage, on the other hand, lies in the effect of the central binary on the thickness of the disk. While it is difficult to compute exactly the effect of tidal distortion of the disk due to the close binary, it seems possible that the disk might be warped or puffed up to a thickness incompatible with the tilted thin-disk model. Kumar (1987) has shown that it is possible to imagine a configuration in which the inner binary twists the disk to a thickness that effectively mimics Huang's (1965) thick-disk model. Kumar (1987) takes the inclination of the close stars to be $\sim 20^{\circ}$ with respect to the orbital plane of the wide system. However, as was already noted, the thick-disk model is apparently ruled out by the observation of the mid-eclipse brightening in the 1982-84 eclipse. Van Hamme \& Wilson (1986), on the other hand, have argued that it is also possible to imagine a configuration with a close binary in which the tidal interactions are sufficiently negligible that a thin disk is still viable. Van Hamme \& Wilson (1986) explored the question by looking at the motion of 
a test particle in the potential of a hypothetical three-body system: a restricted four-body problem. By performing numerical simulations they found that a disk particle would wander only a very small distance (less than $10^{-3}$ of the radial distance) if the inner binary were closely aligned with the wide orbit - within approximately $1^{\circ}$. While this is encouraging, it is not clear that such a small inclination can lead to a disk geometry that will explain the light curve, both by eclipsing enough of the primary and letting enough light through during mideclipse. However, these simulations lead us to believe that the thin disk with central binary is physically viable. It is worth noting that, unlike the high-mass model, the low-mass proposal requires the secondary to be a binary, as explained by Webbink (1985) and Carroll et al. (1990).

Thus we may say that the binary secondary is an attractive model, even though it is plagued with the unresolved problem of the gravitational stability of the thin disk. Once again this proposal, if true, makes $\epsilon$ Aurigae a fascinating system with which to study stellar evolution, as there are only about 26 triple systems known in which all three stars are close enough to interact within their lifetimes (Fekel 1981).

Nevertheless, it is far from clear that the attractiveness of this model makes alternatives unworthy of consideration. One possibility is a suggestion made by Cameron (1971), that a black hole is located at the center of the disk. The motivation for this hypothesis, of course, was the search for an object that could be as massive as 13 to $15 \mathrm{M}_{\odot}$ without being visible, even at ultraviolet wavelengths. In fact, $\epsilon$ Aurigae was included in the survey of black hole candidates by Trimble \& Thorne (1969), who did not accord it serious consideration since there were no observed X-rays or gamma-rays (and there have been none since). However, while such observations would be compelling evidence that a black hole was present, they should not be thought of as necessary to its existence. High-energy radiation would only be prominent in an accreting system, and it is not implausible that this isn't happening in $\epsilon$ Aurigae. (For example, if the black hole had a less massive close companion, accretion would be suppressed by angular momentum transfer to the disk.) Furthermore, it is still possible that X-rays from a disk are beamed away from us or attenuated. Therefore, although the black hole scenario is not absolutely required, it is still a viable (but unlikely) alternative to other possibilities (see §4.7).

\section{Towards Solving the Mystery of $\epsilon$ Aurigae}

\subsection{Accurate Parallax}

It was expected that a definitive trigonometric parallax from Hipparcos would permit the distinction between the high mass (normal F supergiant) and the lowmass model $\left(\sim 1 \mathrm{M}_{\odot}\right.$ post-AGB F supergiant). However, the Hipparcos parallax $\pi(\mathrm{HIPP})=1.60 \mathrm{mas}$ and its relatively large uncertainty of $\pm 1.16 \mathrm{mas}$ lead to a distance range $450 \mathrm{pc}<d<850 \mathrm{pc}$. The resulting absolute magnitudes for the F supergiant are $\mathrm{M}_{V} \sim-6.1 \mathrm{mag}$ to $-7.5 \mathrm{mag}$ (with $\mathrm{A}_{V}=0.84 \mathrm{mag}$ ). Less luminous $\mathrm{M}_{V}$-values, $\mathrm{M}_{V}>-6 \mathrm{mag}$, are appropriate for post AGB stars while values $M_{V}=-6$ to -8 are appropriate for evolved $13-15 M_{\odot}$ FI stars.

Within the next few years it will be possible to measure parallaxes with a precision of better than $\sim 0.2$ mas with the small German astrometric satel- 
lite DIVA (see: www.ari.uni-heidelberg.de/diva/diva.html). $D I V A$ is currently planned to be launched during 2004/05. Even better parallaxes will be possible with NASA's Space Interferometry Mission (SIM), due for launch around 2008. With $S I M$ it will be possible to measure parallaxes with an unprecedented precision of better than 0.05 mas! With high precision parallax determinations possible during the next several years it should be possible to unambiguously decide between the two competing models for $\epsilon$ Aurigae.

\subsection{High Precision Astrometry}

Because of its long period and wide orbit, $\epsilon$ Aurigae is a visual astrometric binary. Conventional photographic astrometry of the system reveals the motion of the FOI star about the system's barycenter. Strand (1959) found a value of the angular semi-major axis of the F-star to be $a_{1} \sin i=14 \pm 4$ mas. On the other hand, the analysis of Sproul Observatory plates by van de Kamp (1978) and later by Heintz \& Cantor (1994) yielded values of $a_{1}=22.7 \pm 1.0$ mas and $22.4 \pm 1.8$ mas, respectively. For example, adopting the absolute semimajor axis ( $a_{1}$ in $\mathrm{km}$ ) for the F0I star from an accurate radial velocity curve results in an independent distance determination. With Wright's (1970) value of $a_{1}=13 \mathrm{AU}$, Heintz \& Cantor's (1994) angular semi-major axis yields a distance $d \sim 600$ pc. It may be fortuitous, but this value is in good agreement with the Hipparcos distance of $625 \pm 250 \mathrm{pc}$.

It will be valuable to carry out a modern astrometric study to determine the astrometric orbital properties of $\epsilon$ Aurigae. The long period presents some challenges but an excellent astrometric orbit could be determined from modern, high-precision astrometry. Analysis of a well defined astrometric orbit of the FO I star could yield independent determinations of its orbital elements, as well as an improved distance.

\subsection{Interferometry}

Recently the F-star's angular diameter of $\alpha=2.23 \pm 0.05$ mas has been measured by Nordgren et al. (2001) using the Navy Prototype Optical Interferometer (NPOI) and the Mark III Optical Interferometer. When this value is combined with the astrometric angular semimajor axis $a_{1}=22.2$ mas from Heintz \& Cantor (1994), the fractional radius $(r)$ of the F-supergiant is $r=R / a=0.05$. When the spectroscopic radial velocity value $a_{1}=13 \mathrm{AU}$ is assumed, the absolute radius of the star is then $R=140 \mathrm{R}_{\odot}$. It is noteworthy that this result is independent of the parallax determination. When the radius of the star is computed directly using the star's interferometric angular diameter and the Hipparcos parallax, the resulting radius of the F-supergiant is $R=150 \mathrm{R}_{\odot}$. The agreement between these two values is very reassuring and indicates that the adopted distance and the computed stellar radius of $R \sim 140-150 \mathrm{R}_{\odot}$ is probably correct. However, this indicates that the value of the F-supergiant's radius of $R \sim 200 \mathrm{R}_{\odot}$ adopted by Carroll et al. (1990) should be revised downward.

\subsection{High Precision Radial Velocity Curve}

The spectroscopic elements for $\epsilon$ Aurigae given by Wright (1970) are based on the photographic-era radial velocities. Currently there are large uncertainties in the spectroscopic values of the orbital eccentricity and the argument of periastron 
that cause the large uncertainty in the eclipse time of the secondary object by the F-supergiant. With the techniques currently available, radial velocities of the F component can be measured with precision of better than $50 \mathrm{~m} / \mathrm{s}$. Care would have to taken to account for the small radial velocity variations arising from the pulsation of the F-supergiant. Because of the 27 year orbital period, this project requires a long term commitment in time and effort.

$\epsilon$ Aurigae is a single line spectroscopic binary but with the increased sensitivity of IR detectors, it might be feasible to measure spectral features of the cool component in the IR and determine its orbital radial velocities. If this could be done successfully, then from the resulting double-line radial velocity curve, the masses of both components could be directly determined and thus eliminate once and for all many of the uncertainties about the nature of the system. High signal to noise IR spectroscopy could be carried out with existing ground-based telescopes such as Keck, or with the Next Generation Space Telescope (NGST), or possibly with the Space Infrared Telescope Facility (SIRTF).

\subsection{Photometry - Long-Term and Eclipse Observations}

Nearly continuous photoelectric photometry of $\epsilon$ Aurigae has been carried out since the last eclipse in 1982-1984. This photometry is being conducted at Fairborn Observatory under the supervision of Louis Boyd using a small robotic telescope equipped with $U B V$ filters. It is hoped that this photometry (as well as photometry carried out elsewhere) will continue up to and beyond the 20092011 eclipse. Outside of primary eclipse the light curve is dominated by the complex low amplitude light variations arising from the presumed pulsations of the F-supergiant. An illustration of these light variations is shown in Fig. 4 where 3 years of $V$-band observations are plotted.

Since the pulsations of a star are closely tied to its mass, radius, and internal structure, it might be possible to distinguish a Pop. I helium shell burning supergiant from a low mass, post-AGB supergiant from their pulsation characteristics. With that in mind Donahue, Boyd and Guinan are studying this possibility by subjecting the photometric $U B V$ data sets of $\epsilon$ Aurigae to period searches. The analysis of the 1987-2002 photometry indicates a complex pattern of periods with a major broad peak at $P=79$ days and two smaller and narrower period peaks at 58 days and 120 days respectively. Longer periods are also possible.

No other F-supergiant, however, has comparable photometric coverage. However, the analysis of 2.5 years of Hipparcos photometry of the post-AGB F2 Ib star, 89 Her, shows remarkably different period characteristics (shorter times scales) than observed for $\epsilon$ Aur. This is interesting, but additional photometry of $\epsilon$ Aur and bonafide Pop. I (massive) supergiants and post-AGB supergiants is necessary before any definite conclusions can be drawn.

It will be important to cover the next eclipse. In particular, observations near the middle of the eclipse (early August 2010) will be critical to determine if the mid-eclipse brightening is again present and to determine its nature. However, $\epsilon$ Aurigae will be a morning star, located low in the August skies near mid-eclipse. Care will have to be taken to secure good photometry during this critical time but it will be worth the effort. 


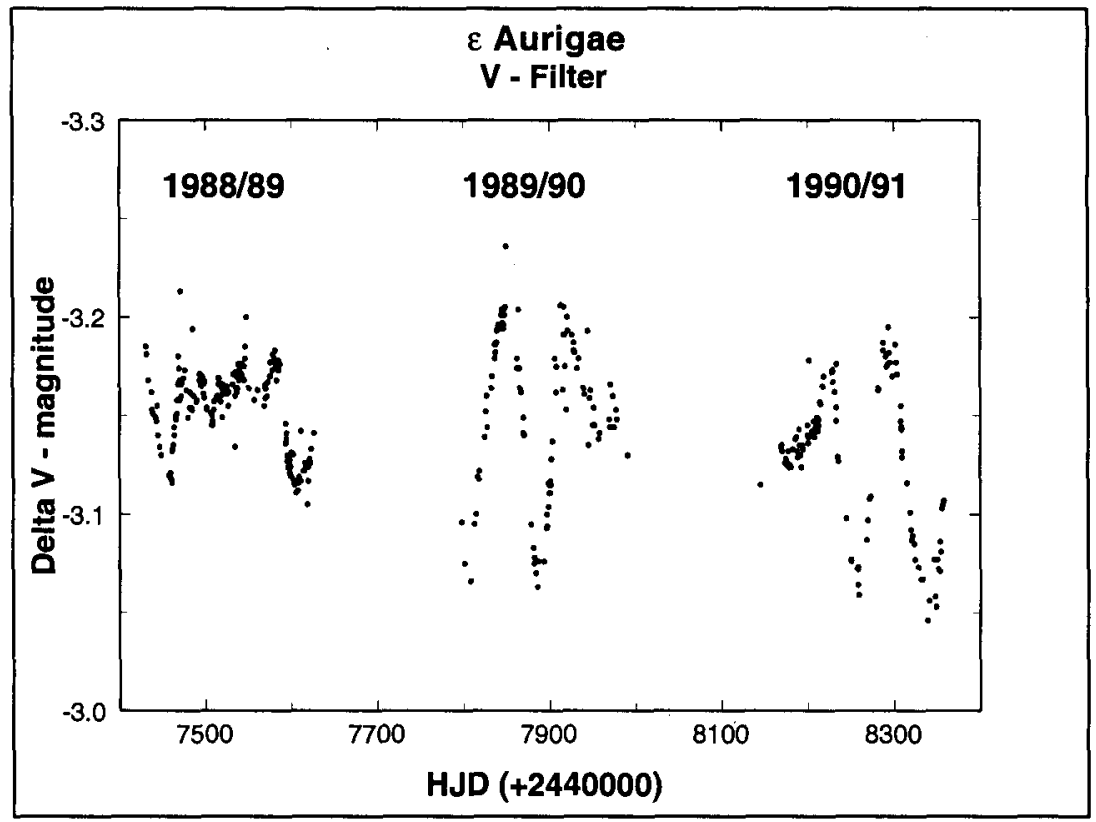

Figure 4. Plot of the differential $V$-mag measures of $\epsilon$ Aurigae obtained by L. Boyd from 1988-1991 at Fairborn Observatory. The changing low-amplitude light variations arising from the F-supergiant are shown. 


\subsection{Imaging the Disk in the Infrared}

Working in the infrared, using adaptive optics or interferometry, it might be possible to actually image the cool disk object. Large infrared excesses have been found for the system that indicate that the cool disk will contribute a significant fraction of the light at IR wavelengths longer than $5 \mu \mathrm{m}$. Depending on the total systemic mass and distance adopted, the angular diameter of the disk object is expected to be relatively large. For example, with $d=625 \mathrm{pc}$, adopting a disk diameter of $\sim 18 \mathrm{AU}$ results in a disk angular diameter of $\alpha$ (disk) $\sim 29$ mas. At greatest orbital elongation, the center of the disk would be located some $\sim 40$ mas from the F0I star. It might be feasible to carry out these measures in the infrared with large aperture telescopes such as Keck, Gemini North using Adaptive Optics or Interferometric techniques.

\subsection{Pointed X-ray Observations}

Both Cameron (1970) and Wilson (1971) suggested that a stellar black hole could be located in the center of the large disk. This idea was advanced to explain the apparent high mass $\left(\sim 13 \mathrm{M}_{\odot}\right)$ and extreme under-luminosity of the object(s) in the center of the disk. However, if a black hole were located at the disk's center, then the strong gravity field of the collapsed object would produce strong X-ray emissions from the inner regions of the accretion disk with X-ray luminosities of $L_{x} \sim 10^{35} \mathrm{erg} / \mathrm{s}$. The strong X-ray emissions observed from high mass X-ray binaries (HMXBs) that contain black hole objects is explained by this mechanism. However, even if $\mathrm{X}$-rays were being emitted by the inner disk, they could be strongly attenuated by the disk itself (seen nearly edge-on) and also by the expected strong stellar winds of the $\mathrm{F}$ supergiant. If the disk contains a relatively massive (non-degenerate) pre-main sequence (PMS) star, then a lower level of X-ray emission $\left(L_{x} \sim 10^{31} \mathrm{erg} / \mathrm{s}\right)$ would be expected. The sources could be distinguished from the tell-tale differences in the X-ray energy distributions of HMXBs (hard X-ray sources) and PMSs (soft X-ray sources).

From all sky X-ray surveys, $\epsilon$ Aur has not been detected as an X-ray source. A re-examination of the ROSAT all sky $\mathrm{X}$-ray observations indicate no significant $\mathrm{X}$-ray source is located at the position of $\epsilon$ Aurigae. As far as we know, there have been no pointed long exposures of $\epsilon$ Aurigae attempted with the more powerful X-ray satellites Chandra and XMM. Deep X-ray observations of $\epsilon$ Aurigae would, however, be useful in constraining (or even detecting) the object (or objects) concealed in the center of the accretion disk.

\subsection{Far Ultraviolet Observations}

The first ultraviolet (UV) and far ultraviolet (FUV) observations of $\epsilon$ Aurigae were obtained with the International Ultraviolet Explorer (IUE) satellite starting a few years before the 1982-1984 eclipse. These observations were primarily made at low dispersion and cover wavelengths of $1170-3200 \AA$. Several papers have been published based on IUE spectroscopy (e.g. Stencel 1985). IUE spectroscopy taken during the 1982-1984 eclipse indicates that FUV eclipse depth at wavelengths $<1400 \AA$ may be shallower than observed at longer wavelengths. At face value this effect is evidence for FUV emission from the eclipsing object (the disk). Additionally, IUE observations (made both during and outside the eclipse) indicate excess FUV fluxes over those expected from the F0 supergiant 
(see Parthasarathy \& Lambert 1983). However, the interpretations of the FUV $I U E$ spectra may have been compromised (in part) by the effects of scattered light.

In spite of the intriguing questions raised by the IUE/FUV observations of $\epsilon$ Aurigae, only a single epoch UV spectrum has been reported with the much more powerful Hubble Space Telescope (HST). The HST observations were carried out by Sheffer \& Lambert (1999) with the Goddard High Resolution Spectrograph (GHRS) during February 1996. The HST observations were secured at an orbital phase when the secondary (disk) was orbiting toward the expected secondary eclipse by the F0I star. The GHRS spectrum covers the wavelengths between $1175-1460 \AA$ and is free from scattered light. The spectrum contains numerous emission and absorption lines. Sheffer \& Lambert (1999) interpreted the observed double-peaked emissions as arising from the rotating inner edge of the secondary disk. The radial velocity measures seem to favor the high mass model for the system.

It's a pity that more FUV spectra of $\epsilon$ Aurigae were not obtained with $H S T$ at different orbital phases. We are sure this was not for the lack of $H S T$ proposals. None the less it is not too late to secure medium or high resolution FUV spectra with $H S T$ (now with STIS). Another high quality FUV spectrum might be sufficient to determine the radial velocity of the secondary object that could lead to a mass determination of the system. FUV/UV spectroscopy of $\epsilon$ Aurigae with HST could be done efficiently as part of the HST Snapshot program. Currently NASA is supporting HST at least until 2010.

It would be very interesting to observe at shorter wavelengths than possible with $H S T$ with the hope of identifying unambiguously the source of the excess FUV emission. Medium resolution FUV spectroscopy $(920-1190 \AA)$ of $\epsilon \mathrm{Au}$ rigae with the Far Ultraviolet Spectroscopic Explorer (FUSE) satellite could be extremely valuable for this experiment.

\subsection{Radio Observations}

$\epsilon$ Aurigae was detected at $250 \mathrm{GHz}(\lambda=0.1 \mathrm{~cm})$ as a radio source with an observed flux of $S=9 \pm 2 \mathrm{mJy}$ (Altenhoff et al. 1994). The expected $250 \mathrm{GHz}$ flux for the star from the Raleigh-Jeans relation is $S(\mathrm{~F} 0 \mathrm{I}) \sim 2 \mathrm{mJy}$. This value was computed using $T_{\text {eff }}=7800 \mathrm{~K}$ for the F0I star and the recently measured angular diameter of the F0I star of $\alpha=2.23$ mas from the NPOI by Nordgren et al. (2001). So it appears that the most of the radio emission originates from the secondary disk companion. It will be important to obtain additional radio observations to search for variability. With the $V L A$ or $V L B I$ radio arrays it might be possible to map the astrometric orbit of the disk object. This could lead to the determination of its mass.

\subsection{The First Eclipse of the New Millennium}

The next primary eclipse of $\epsilon$ Aurigae occurs during 2009-2011. The eclipse is expected to begin during June/July 2009 (1st contact) and end (4th contact) nearly two years later during April/May 2011. The 2nd and 3rd contacts of the eclipse should occur during November 2009 and January 2011, respectively. The expected time of mid eclipse is early August, 2010. Many instrumental and technical advances have been made since the 1982/84 eclipse and it should be very 
interesting to see what new discoveries, as well as new problems, are uncovered from ground and space based telescopes. Full wavelength coverage from X-ray to radio should be possible with an array of powerful new instruments. Adaptive optics imaging, interferometry, ultra-high signal-to-noise and high spectral resolution spectroscopy, high precision photometry should also be available. Also, within the next few years global networks of robotic telescopes should be on-line for continuous photometry. Thus, during the first eclipse of the millennium, now only several years away, an astronomical armada of powerful instruments will be available. These should hopefully lead to the "demystification" of this intriguing star.

\section{Some Concluding Remarks}

$\epsilon$ Aurigae presents observational and theoretical stellar astrophysicists with unique opportunities and daunting challenges. At present, our knowledge of the mass and luminosity of the system is too uncertain to pinpoint its evolutionary state. The two viable options are the high-mass model in which the primary is in a helium shell burning phase, recently off the main sequence, and the low-mass model in which the primary is a post-AGB star and PPN object contracting to a white dwarf state. In the first model, the large disk around the secondary is likely to represent a protoplanetary system at an age of around $10^{7}$ years. The low-mass model explains the disk as the result of recent mass transfer from the primary; in this case, the primary star is of significant interest to stellar evolution studies since it is in such a rare stage of rapid evolution.

The question of the object at the center of the disk remains unresolved. While it is difficult to imagine a single star lurking there unobserved, there are uncomfortable aspects of a binary model or a hidden black hole in the disk's center. The necessity of further study is evident, including the search for a secondary eclipse in the infrared and continual monitoring of the star in all wavelengths. Hopefully after the next eclipse during 2009-2011, with large telescopes operating on the ground and in space, adaptive optics and interferometry, new generations of sensitive detectors at all wavelengths, we will be lucky enough to witness the unraveling of the mystery of this perplexing system. Let's all hope (and pray) we are here to participate and enjoy this special 27 year astronomical event. Coordinating the observations of the next eclipse are starting to be organized. For more information on the international $\epsilon$ Aurigae campaign, contact Dr. Robert Stencel at the University of Denver (rstensel@du.edu).

This research is partially supported by NSF/RUI grant AST-0071260 which we gratefully acknowledge.

\section{References}

Allenhoff, W. J., Thum, C., \& Wendker, H. J. 1994, A\&A, 281, 161

Backman, D. E., Beckfin, E. E., Cruikshank, D. P., Joyce, R. R., Simon, T., \& Tokunaga, A. 1984, ApJ, 284, 799

Backman, D. E., Witteborn, P. C., \& Gillett, F. C. 1992, ApJ, 385, 670

Bailey, F. 1843, MmRAS, 13, 1 
Boehm, C., Ferluga, S., \& Hack, M. 1984, A\&A, 130, 419

Bond, H. E., Carney, B. W., \& Grauer, A. D. 1984, PASP, 96, 176

Cameron, A. G. W. 1971, Nature, 229, 178

Carroll, S. M., Guinan, E. F., McCook, G. P., \& Donahue, R. 1990, ApJ, 367, 278

Castelli, F. 1978, A\&A, 69, 23

Drilling, J. S., \& Landolt, A. U. 2000, in Allen's Astrophysical Quantities (4th edition), ed. A. N. Cox (New York: Springer-Verlag), 389

Eggleton, P. P., \& Pringle, J. E. 1985, ApJ, 288, 275

Ferluga, S. 1990, A\&A, 238, 270

Flammarion, C. 1882, Les Etoiles et les Curiosités du Ciel, Suppl. Astronomie Populaire (Paris: Marpon \& Flammarion)

Fekel, F. C., Jr. 1981, ApJ, 246, 879

Gonzalez, G., Lambert, D. L., Wallerstein, G., Rao, N. K., Smith, V. V., \& McCarthy, J. K. 1998, ApJS, 114, 133

Gyldenkerne, K. 1970, Vistas Astron., 12, 199

Hack, M. 1961, Mem. Soc. Astr. Ital., 32, 351

Hartigan, P., Hartmann, L., Kenyon, S., Strom, S., \& Skrutskie, M. 1990, ApJ, $354, \mathrm{~L} 25$

Heintz, W. D., \& Cantor, B. A. 1994, PASP, 106, 363

Herbig, G. H., \& Boyarchuk, A. A. 1968, ApJ, 153, 397

Hrivnak, B. J., \& Milone, E. F. 1984, ApJ, 282, 748

Hrivnak, B. J., Kwok, S., \& Volk, K. M. 1989, ApJ, 346, 265

Huang, S. -S. 1965, ApJ, 141, 976

Iben, I. 1967, ARA\&A, 5, 571

Iben, I. 1982, ApJ, 260, 821

Kerber, F., Koppen, J., Roth, M., \& Trager, S. C. 1999, A\&A, 344, L79

Knobel, E. B. 1917, Ulugh Beg's Catalogue of Stars (Washington D.C.: The Carnegie Institution)

Kopal, Z. 1971, Ap\&SS, 10, 332

Kuiper, G. P., Struve, O., \& Strömgren, B. 1937, ApJ, 86, 570

Kumar, S. 1987, MNRAS, 225, 823

Lamb, S. A., Iben, I. I., \& Howard, W. M. 1976, ApJ, 207, 209

Larson, R. B. 1978, in Protostars and Planets, ed. T. Gehrels (Tucson: Univ. Arizona Press), 43

Lawlor, T. M., \& MacDonald, J. 2002, Ap\&SS, 279, 123

Lissauer, J. J., \& Backman, D. E. 1984, ApJ, 286, L39

Lissauer, J. J., Wolk, S. J., Griffith, C. A., \& Backman, D. E. 1996, ApJ, 465, 371

Morris, S. C. 1962, JRASC, 56, 210

Nordgren, T. E., Sudol, J. J., \& Mozurkewich, D. 2001, AJ, 122, 2707

Osmer, P. S. 1972, ApJS, 24, 247 
Paresce, F., \& Burrows, Ch. 1987, ApJ, 319, L23

Parthasarathy, M., \& Lambert, D. L. 1983, PASP, 95, 1012

Pendleton, Y. J., \& Black, D. C. 1983, AJ, 88, 1415

Peters, C. H. F., \& Knobel, E. B. 1915, Ptolemy's Catalogue of Stars: A Revision of the Almagest (Washington D.C.: The Carnegie Institution)

Pringle, J. E. 1981, ARA\&A, 19, 137

Saito, M., \& Kitamura, M. 1986, Ap\&SS, 122, 387

Schönberner, D. 1983, ApJ, 272, 708

Sheffer, Y., \& Lambert, D. L. 1999, PASP, 111, 829

Skrutskie, M. F., Dutkevich, D., Strom, S. E., Edwards, S., Strom, K. M., \& Shure, M. A. 1990, AJ, 99, 1187

Smith, B. A., \& Terrile, R. J. 1984, Science, 226, 1421

Snell, R. L, \& Edwards, S. 1981, ApJS. 45, 121

Stencel, R. E. 1985, The Recent Eclipse of Epsilon Aurigae, NASA Conf. Publ. 2384

Strand, K. A. 1959, AJ, 64, 346

Strom, K. M., Strom, S. E., Edwards, S., Cabrit, S., \& Skrutskie, M. 1989a, AJ, 97, 1451

Strom, S. E., Edwards, S., \& Strom, K. M. 1989b, in The Formation and Evolution of Planetary Systems, ed. H. A. Weaver \& L. Danly (Cambridge: Cambridge University Press), 91

Taranova, O. G., \& Shenavrin, V. I. 2001, Astron. Lett., 27, 338

Trimble, V. L., \& Thorne, K. S. 1969, ApJ, 156, 1013

van de Kamp, P. 1978, AJ, 83, 975

Van Hamme, W., \& Wilson, R. E. 1986, ApJ, 306, L33

Webbink, R. F. 1985, in The 1982-84 Eclipse of Epsilon Aurigae, ed. R. Stencel (Washington: NASA), 49

Wilson, R. E. 1971, ApJ, 170, 529

Wright, K. O. 1970, Vistas Astron., 12, 147 